\title{
Comparison and Evaluation of the Low-Level Laser and the Red and Blue LED Effects on Wound Healing in Rabbit
}

\author{
Marjan Ghaemi ${ }^{*}$, Davood Sharifi ${ }^{2}$, Soheila Mokmelii ${ }^{3}$, Golshad Kowsari ${ }^{4}$, Pejman Mortazavi ${ }^{5}$, Pouneh Golmai ${ }^{6}$ \\ ${ }^{1}$ Yas Hospital, Tehran University of Medical Science, Tehran, Iran \\ ${ }^{2}$ Department of Surgery and Radiology, Faculty of Veterinary Medicine, the University of Tehran, Tehran, Iran \\ ${ }^{3}$ Canadian Laser Training Institute (CLTI), Victoria, Canada \\ ${ }^{4}$ Faculty of Veterinary Medicine, University of Tehran, Tehran, Iran \\ ${ }^{5}$ Department of Veterinary Pathology, Faculty of Specialized Veterinary Sciences, Science and Research Branch, Islamic \\ Azad University, Tehran, Iran \\ ${ }^{6}$ Department of Internal Medicine, Lenox Hill Hospital, New York, USA
}

\section{*Correspondence to \\ Marjan Ghaemi, Yas Hospital Tehran University of Medical Science, Tehran, Iran. North Nejatollahi St, Karim Khan Ave. Post Code: 1598718311 Tel: +989121967735; Email: Marjan_ghaemi@yahoo. com}

Published online July 6, 2019

\begin{abstract}
Introduction: Wound healing is a dynamic, interactive process to achieve the restoration of skin integrity and proper function after damage. Applying a low-level laser (LLL) and light emitting diodes (henceforth LEDs) is introduced in previous studies to accelerate the process of wound healing. The aim of this study is to compare the effect of the LLL and LEDs on wound healing in rabbits.

Methods: Full thickness same size square excision wounds were created on the dorsum of the rabbits. Twenty rabbits were randomly divided into four groups, according to the treatment received. Group 1: the AIGallnP (aluminium gallium indium phosphide) laser $\left(4 \mathrm{~J} / \mathrm{cm}^{2}\right.$ ); group 2: the red LED $\left(30 \mathrm{~J} / \mathrm{cm}^{2}\right)$; group 3: the blue LED $\left(60 \mathrm{~J} / \mathrm{cm}^{2}\right)$ and group 4, as the control group, was not irradiated. After 30 days, the wounds were evaluated both morphologically and histopathologically. Statistical significance was defined as a $P$ value of less than 0.05 .

Results: All interfering methods including the LLL and LEDs had better outcome compared with the control group of both sizes and histopathologic features. The red laser group showed better results compared to the control group and either the LED groups. Comparing LEDs, the red LED performed better than the blue LED.

Conclusion: This study confirmed the significant effects of the LLL and LEDs on wound healing. Comparing the LLL and LED, the LED may be a better choice, especially for bedridden or debilitated patients. The LED may also more cost effective in wound healing in comparison with the LLL.

Keywords: Light emitting diode; Low-level laser; Wound healing.
\end{abstract}

\section{Introduction}

Wound repair is a dynamic, interactive process involving different cell types, extracellular matrix, and growth factors to achieve the restoration of skin integrity and proper function after damage.

Low-level laser therapy (LLLT) as a therapeutic modality in wound healing was introduced by Mester and colleagues via the application of a low-level Ruby laser..$^{2-4}$ LLLT proved to have beneficial effects on rat skin wound repair, leading to earlier regression of the inflammatory phase, faster re-epithelialisation, and acceleration in the maturation phase of healing. ${ }^{5}$

Light emitting diodes (LEDs) are small, robust devices that emit a narrow band of electromagnetic radiation ranging from ultraviolet to the visible and infrared wavelengths. LEDs usually generate low-intensity light that can be configured on small chips or connected to small lamps. ${ }^{10-12}$

Klebanov et al compared lasers and LEDs and found that they both had very close effects on wound healing. ${ }^{6}$ But Lasers have limitation to produce the efficient wavelength combination optimal for wound healing. In contrast, LEDs allow the control of spectral composition and may be arranged in flat arrays of all sizes for the treatment of either small or large areas. LEDs offer an effective alternative to the use of conventional light sources.

The aim of this study is to compare the effect of the LLL and the LED on wound healing in rabbits. Recent studies revealed that the LEDs, compared to the LLL, bring about effects on improving wound healing and have low cost

Please cite this article as follows: Ghaemi M, Sharifi D, Mokmeli S, Kowsari G, Mortazavi P, Golmai P. Comparison and evaluation of the low-level laser and the red and blue LED effects on wound healing in rabbit. / Lasers Med Sci. 2019;10(3):189-193. doi:10.15171/ jlms.2019.30. 
and easy handling. The results of this study may help the clinicians to use the LEDs or the LLL based on their patient's condition.

\section{Methods}

Twenty adult male rabbits in the same age and weight groups were randomly divided into four equal groups of 5 animals each, according to the treatment received. The AlGalInP (aluminium gallium indium phosphide) laser was applied to the first group. In the second and third groups, the red and blue LEDs were used with a dose of $0.6 \mathrm{~J} / \mathrm{cm}^{2}$. Group 4 was not irradiated.

\section{Surgery Procedure}

The rabbits were initially anaesthetized and subsequently shaved, and under sterile conditions, six full thickness excision wounds with the size of $1 \mathrm{~cm}^{2}$ were created on the dorsum of each rabbit. The depth of the wounds was standardized by reference to detection of the muscle plane. Bandages were routinely replaced on days 3 and 5, or when deemed necessary.

\section{Phototherapy}

The irradiation started immediately after surgery on either the left or right side of the body and was repeated every other day for 30 days. The low-level AlGalInP laser at a wavelength of $650 \mathrm{~nm}$ (nanometer) was applied with an output power of $10 \mathrm{~mW}$ and a dose of $1 \mathrm{~J} / \mathrm{cm}^{2}$ in 100 seconds for each wound. The red LED (designed by Canadian Optic and Laser Production Center) with a wavelength of $630-680 \mathrm{~nm}$ and $75 \mathrm{~mW}$ power was exposed with a dose of $1 \mathrm{~J} / \mathrm{cm}^{2}$ for 200 seconds. In the third group, the blue LED (designed by Canadian optic and laser production center) with the wavelength of 450$470 \mathrm{~nm}$ and power of $75 \mathrm{~mW}$ with a dose of $1 \mathrm{~J} / \mathrm{cm}^{2}$ in 200 seconds was used.

Although the radiation dose for the laser group and the LED groups was the same, because of a different design, the power density of the applied laser was $0.01 \mathrm{~W} / \mathrm{cm}^{2}$ compared to that of the LEDs, which was $0.005 \mathrm{~W} / \mathrm{cm}^{2}$.

Photography

The wounds were photographed using a digital camera after surgery and on days 3, 7, 14, 21 and 30 after surgery. Samples were evaluated daily and the wound extent was measured by a digital caliper.

\section{Histopathology}

Thirty days after surgery, the specimens, including the entire wound plus a portion of the wound margin and subcutaneous tissue, were removed for histomorphologic and histophotometric examinations to evaluate wound healing. Changes in the number of the polymorphonuclear inflammatory cells, the degree of edema, the mononuclear inflammatory cells, and collagen fiber deposition were evaluated.

The means of ulcer sizes were compared among four groups on day 30 using ANOVA. Three pair-wise comparisons were made between the laser group and the two LED groups using $t$ test with Bonferroni correction for $P$ value, comparing $t$ test statistics to 0.0167 for significance.

All experimental procedures were performed under the conditions described in "The manual for the care and use of laboratory animals" and adopted by the Faculty of Veterinary Medicine of University of Tehran, Tehran, Iran.

\section{Results}

Macroscopic Observation

Wound healing was completed in all rabbits within the 30-day observation period. The wounds were measured on days $0,3,7,14,21$ and 30 . Table 1 shows the time course of changes in the relative wound area. The baseline (day zero) wound contraction result was alike across the four groups under study $(P=1.000)$.

Wound healing between the four groups was compared using two-way ANOVA. Comparing the size of ulcers among the 4 groups on day 30 showed a statistically significant difference between the interventions in the LED and Laser groups and no intervention in the control group (Table 2, $P<0.001$ ). A series of pair-wise comparisons further indicated the Laser irradiation as the most effective treatment and better than both the Blue $(P<0.001)$ and $\operatorname{Red}(P<0.001)$ LED irradiation. Although ulcers in the Red LED group showed a tendency to heal

Table 1. Comparison of Wound Contraction in 4 Groups

\begin{tabular}{|c|c|c|c|c|c|c|c|c|c|c|c|c|}
\hline Group & & $\mathbf{0}$ & 3 & 6 & 9 & 12 & 15 & 18 & 21 & 24 & 27 & 30 \\
\hline \multirow{2}{*}{ Control } & Mean & 20.00 & 20.00 & 18.07 & 18.07 & 17.07 & 16.05 & 14.95 & 13.15 & 13.07 & 10.90 & 8.92 \\
\hline & Standard error of mean & 0.00 & 0.00 & 0.64 & 0.64 & 0.75 & 0.45 & 0.58 & 0.72 & 0.74 & 0.58 & 0.33 \\
\hline \multirow{2}{*}{ Blue LED } & Mean & 20.00 & 19.25 & 18.97 & 18.92 & 18.00 & 17.07 & 16.07 & 13.95 & 10.10 & 8.05 & 4.97 \\
\hline & Standard error of mean & 0.00 & 0.31 & 0.26 & 0.25 & 0.15 & 0.06 & 0.41 & 1.04 & 0.39 & 0.30 & 0.32 \\
\hline \multirow{2}{*}{ Red LED } & Mean & 20.00 & 19.07 & 18.07 & 17.35 & 16.97 & 13.92 & 12.00 & 10.04 & 8.00 & 4.95 & 4.05 \\
\hline & Standard error of mean & 0.00 & 0.22 & 0.21 & 0.21 & 0.26 & 0.26 & 0.09 & 0.33 & 0.18 & 0.16 & 0.06 \\
\hline \multirow{2}{*}{ Laser } & Mean & 20.00 & 18.00 & 17.00 & 16.00 & 14.02 & 12.05 & 9.97 & 8.05 & 5.97 & 4.05 & 1.97 \\
\hline & Standard error of mean & 0.00 & 0.24 & 0.45 & 0.47 & 0.28 & 0.37 & 0.06 & 0.17 & 0.21 & 0.23 & 0.07 \\
\hline
\end{tabular}


Table 2. Mean Ulcer Size on Day 30 and Comparison Between the 4 Groups Using Two-Way ANOVA

\begin{tabular}{lcccc}
\hline Intervention & $\begin{array}{c}\text { Mean Ulcer Size at } \\
\text { Day 30 }\end{array}$ & $\begin{array}{c}\text { ANOVA Coefficients }(\boldsymbol{P}), \\
\text { Compared With Control }\end{array}$ & Difference $(\boldsymbol{P})$, Pair-Wise Comparisons \\
\cline { 4 - 5 } Control & 8.9 & Reference & NA & Laser vs. Red LED \\
\hline Blue LED & 4.9 & $-3.95(<0.001)$ & $0.93(0.031)$ & NA \\
Red LED & 4.1 & $-4.88(<0.001)$ & $0.93(0.031)$ & $3.0(<0.001)$ \\
Laser & 1.9 & $-6.95(<0.001)$ & & $2.08(<0.001)$ \\
\hline
\end{tabular}

$\mathrm{Na}$, Not applicable.

${ }^{a}$ Compare $\mathrm{p}$ to 0.05 ; b Compare $P$ to 0.0167

better than the ones in the Blue LED group, there was no statistically significant difference between these two interventions in terms of mean size of ulcers on day 30 $(P=0.031)$. Also, the repeated-measure ANOVA was done and the results for inter-group analysis were statistically significant $(P=0.0001)$ with a significant difference between the control group and all the other three groups. But in intra-group analysis, there was no significant result in the control group $(P>0.05)$, and in all other groups, there was a significant trend $(P=0.0001)$.

\section{Histopathologic Results}

In the histological sections stained with hematoxylin and eosin, corresponding to the third day after surgical procedure, accentuated edema and marked inflammatory infiltration of predominantly polymorphonuclear cells were observed in the non-irradiated group. However, the laser-irradiated animals exhibited much less polymorphonuclear cells than those in the control group. On the seventh day, it was observed that all irradiated animals exhibited granulation tissue, with angiogenesis and increased mononuclear cell infiltrate, especially macrophages and lymphocytes. The difference was even greater on the 10th day.

The red laser therapy showed better results compared to the control group or either of the LED groups. Comparing LEDs, the red LED performed better compared to the blue LED. In the low-level red laser group, the epidermis was fully restored after 30 days and fibrous tissue was observed in the dermis. Inflammatory reaction with mononuclear and polymorphonuclear was detected while skin appendices were not seen. Some of thick collagen fibers and fibroblasts could be seen in significant amounts (Figure 1).

In group 2 that received the red LED, the epidermal formation of two sides was in the process of completion after 30 days, but still a large amount of scar tissue could be seen. Severe inflammatory reaction was detected in the wound along with fibrous tissue in the dermis with numerous fibroblasts and myofibroblasts, but no appendices were observed (Figure 2).

In group 3, blue LED irradiation scar tissue and hemorrhage were observed and the surface layer of skin was partially formed. Vascular fibrosis in the dermis was observed well. The thickness of the collagen fibers was noticeable. Specific inflammatory reaction was not observed. Fibroblasts and myofibroblasts in the skin and appendices were not seen (Figures 3 and 4).

In the control group, a significant scar was observed 30 days after injury. Epidermis and dermis were seen as partially formed buds with polymorphonuclear inflammatory reaction. Numerous new fibroblasts were seen in the specimens (Figures 5 and 6).

\section{Discussion}

Cutaneous wound healing requires a well-organized integration of the molecular and cellular mechanisms including infiltration of the cells and proliferation,

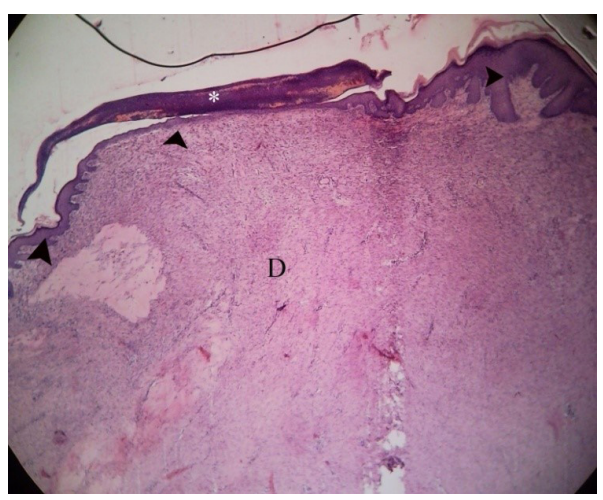

Figure 1. Complete epidermal (arrowhead) formed with a small cottage on it $(*)$ is seen in the fibrous tissue under the dermis (D) in the low-level red laser group.

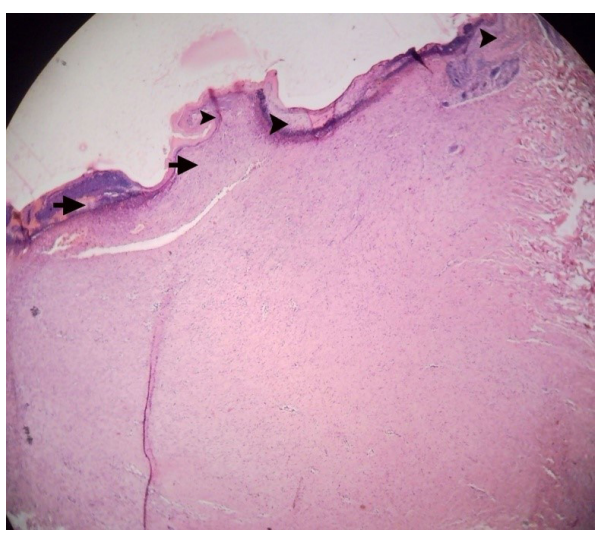

Figure 2. Partially Formed Epidermis (arrowheads) and Fibrous Tissue (arrows) in the Red LED Group. 


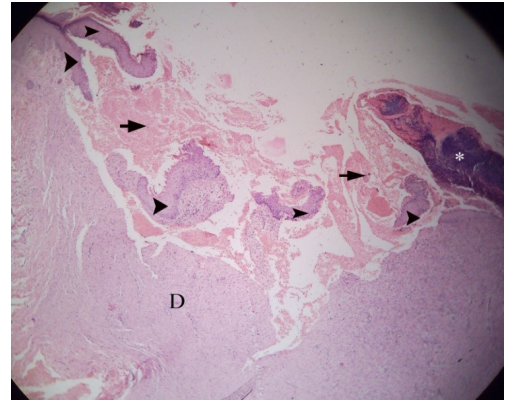

Figure 3. Formation of the new epidermis (arrowhead) surrounding the hemorrhage (arrows), fibrous tissue in the dermis (D) is seen in the blue LED group.

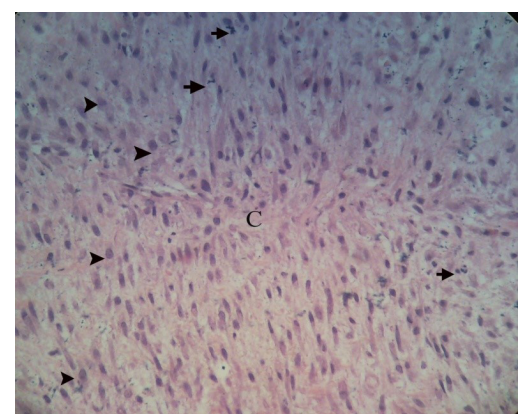

Figure 4. Connective Tissue Filled With Myofibroblast Cells (arrowhead), Collagen Fibers (C) and Inflammatory Reaction (arrows) in the Blue LED Group.

extracellular matrix deposition, and remodeling. The impaired healing process may be the reason for many intrinsic factors including blood supply, angiogenesis, and matrix turnover and extrinsic factors such as infection or trauma. ${ }^{7}$ Therefore, it is reasonable that any factors affecting this process may change the nature of wound healing. Regarding the reviewed studies, LLLT and LED therapy may be effective in wound healing with different mechanisms that have been known until now. ${ }^{2-4}$

In the present investigation, the animals with experimental skin ulcers that received laser treatment presented a statistically significant reduction in the amount of edema and the degree of polymorphonuclear 3-7 days following laser application. Similar findings have been described in the literature. Pugliese et $\mathrm{al}^{8}$ found that experimentally produced wounds in rats exhibited more collagen deposition when irradiated with a low-level laser, compared to the non-irradiated controls. These results were supported by a similar study performed by Bisht and colleagues who investigated the effect of $\mathrm{HeNe}$ laser at $4 \mathrm{~J} / \mathrm{cm}^{2}$ on open skin wounds in rats. ${ }^{9}$

Recently, the LEDs have been presented as a comfortable, potentially highly selected light source for wound healing, regeneration and acne therapy. ${ }^{8}$ The price of the LEDs is much cheaper than lasers and their applications are more convenient.

Many authors of clinical studies have reported the

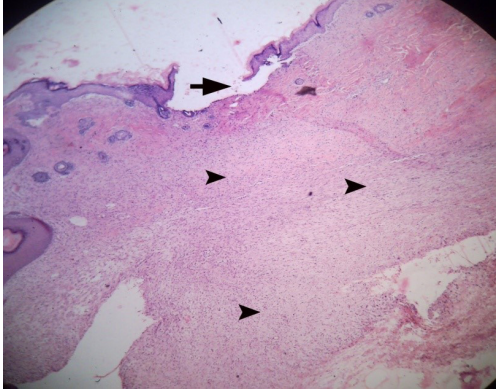

Figure 5. Scar formation and lack of epidermal cells (arrows) in dermal tissue buds broiler area (arrowhead) are seen in the control group.

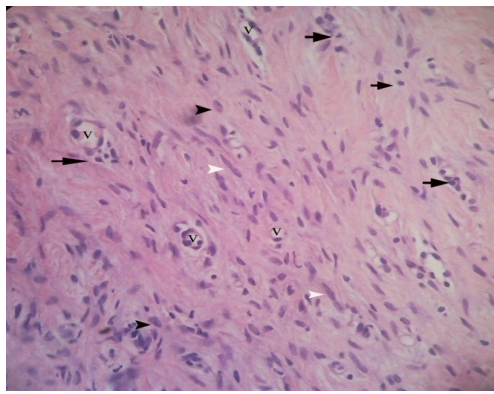

Figure 6. Vascular Sprouts (V) and Fibroblasts (white arrowhead) and Myofibroblasts (black arrowhead) Along With Inflammatory Reaction (arrows) in the Control Group

benefits of LLLT for tissue healing, but the results are inconsistent across studies as others have shown no effect. ${ }^{10-13}$ The LED's effect on wound healing by reducing ulcer size in diabetic cases has been shown to be impressive. ${ }^{12}$

These conflicting results are likely due to variations in treatment factors and limitations in experimental designs, including a comparison of heterogeneous clinical wounds, lack of control groups, and limited or no blinding of investigators. ${ }^{10-14}$

In this study, although the radiation dose for all groups was the same, there were different parameters like different power density, coherency, and wavelength which could affect the final results.

To summarize, the red and blue light emitted by the LED proved to have positive effects on wound healing parameters. One of the mechanisms may be the influence of the blue light on nitric oxide (NO) metabolism. The blue light from the LED also facilitated the recovery of mitochondria inhibited by NO gas through the release of NO from mitochondrial complexes. ${ }^{6}$ Since NO is an important mediator in wound healing and activation of growth factors, it may induce endothelial cell migration, adhesion, and proliferation. ${ }^{15}$

This study showed that both LLL and LED have a significant effect on wound healing. Regarding the comparison between the LED and the laser, it is good to know that the application of the LED is cheaper than that of the laser. Moreover, the LEDs are portable, safer and 
can be used at home; therefore, they are a better choice for the people who are bed-ridden or could not attend clinics for their follow-up remedy.

As laser therapy is in most cases expensive and needs multiple sessions to show its desirable effects, many patients with chronic wounds, especially in less developed countries, may not be able to afford its cost, and LED could prove to be a more economical alternative for them.

It can be concluded that regardless of the better LLLT application in comparison with the LED, it may be a better choice for disabled patients that need to stay at home. A portable LED is also a better choice for personal application compared to LLLT for patients.

\section{Ethical Considerations}

This study have been approved by ethical committee of Shahid Beheshti university of medical sciences (date of approval 30 May 2010).

\section{Conflict of Interests}

The authors declare no conflict of interest.

\section{Acknowledgment}

This research was funded by the grant of the Research Center of Laser Usage in Medicine (RCLUM) of Shahid Beheshti Medical University, Tehran, Iran. This study was accepted for oral presentation in the LASER HELSINKI 2012 International Congress 24 -29 August 2012, Helsinki, Finland

\section{References}

1. Singer AJ, Clark RA. Cutaneous wound healing. $N$ Engl J Med. 1999;341(10):738-746. doi:10.1056/ nejm199909023411006

2. Mester E, Korenyi-Both A, Spiry T, Tisza S. The effect of laser irradiation on the regeneration of muscle fibers (preliminary report). Z Exp Chir. 1975;8(4):258-262.

3. Mester E, Mester AF, Mester A. The biomedical effects of laser application. Lasers Surg Med. 1985;5(1):31-39.

4. Mester E, Spiry T, Szende B, Tota JG. Effect of laser rays on wound healing. Am J Surg. 1971;122(4):532-535.

5. Ribeiro MS, Da Silva Dde F, De Araujo CE, et al. Effects of low-intensity polarized visible laser radiation on skin burns: a light microscopy study. J Clin Laser Med Surg. 2004;22(1):59-66. doi:10.1089/104454704773660994

6. Klebanov GI, Shuraeva N, Chichuk TV, et al. [A comparative study of the effects of laser and light-emitting diode irradiation on the wound healing and functional activity of wound exudate leukocytes]. Biofizika. 2005;50(6):1137-1144.

7. Cavanagh PR, Lipsky BA, Bradbury AW, Botek G. Treatment for diabetic foot ulcers. Lancet. 2005;366(9498):1725-1735. doi:10.1016/s0140-6736(05)67699-4

8. Thomas GN, Tomlinson B, Critchley JA. Guidelines for healthy weight. N Engl J Med. 1999;341(27):2097; author reply 2098. doi:10.1056/nejm199912303412715

9. MauryE, Vassal T, Offenstadt G. Cardiaccontractility during severe ketoacidosis. N Engl J Med. 1999;341(25):1938. doi:10.1056/nejm199912163412516

10. Franklin DJ. Back to the basics. N Engl J Med. 1999;341(27):2099-2100.

11. Sharma OP. Back to the basics. $N$ Engl $J$ Med. 1999;341(27):2099. doi:10.1056/nejm199912303412717

12. Pryde PG, Mittendorf R. The control of labor. $N$ Engl J Med. 1999;341(27):2098-2099. doi:10.1056/ nejm199912303412716

13. Davis GJ. Guidelines for healthy weight. N Engl J Med. 1999;341(27):2097-2098.

14. Wilson WL. Guidelines for healthy weight. N Engl J Med. 1999;341(27):2097; author reply 2098.

15. Campion EW. Notification about early-release articles. $N$ Engl J Med. 1999;341(27):2085. doi:10.1056/ nejm199912303412709 